\title{
Du dilemme des filles et de leurs réserves de vie
}

La crise sorcellaire dans la migration nigériane

On the Predicament of the Daughters and the Resources of Life. Witchcraft Crisis in Nigerian Migration

\section{Simona Taliani}

\section{OpenEdition}

\section{Journals}

\section{Édition électronique}

URL : http://journals.openedition.org/etudesafricaines/22438

DOI : $10.4000 /$ etudesafricaines.22438

ISSN : $1777-5353$

\section{Éditeur}

Éditions de l'EHESS

\section{Édition imprimée}

Date de publication : 15 décembre 2018

Pagination : 737-761

ISBN : 978-2-7132-2743-1

ISSN : 0008-0055

\section{Référence électronique}

Simona Taliani, « Du dilemme des filles et de leurs réserves de vie », Cahiers d'études africaines [En ligne], 231-232 | 2018, mis en ligne le 15 décembre 2020, consulté le 02 mars 2021. URL : http:// journals.openedition.org/etudesafricaines/22438; DOI : https://doi.org/10.4000/etudesafricaines. 22438 


\title{
Du dilemme des filles et de leurs réserves de vie
}

\section{La crise sorcellaire dans la migration nigériane}

\begin{abstract}
«There's been too much attribution of power to the effect of colonialism on our consciousness [...]. [A] true invasion takes place not when a society has been taken over by another society in terms of its infrastructure, but in terms of its mind and its dreams and its myths, and its perception of reality.

If the perception of reality has not been fundamentally, internally altered, then the experience itself is just transitional. There are certain areas of the African consciousness which will remain inviolate. Because the world-view it is that makes a people survive» Ben Okri (Wilkinson 1992 : 86), As Above, So Below ${ }^{1}$.
\end{abstract}

Je me propose dans cet article d'approfondir l'un des aspects de l'organisation clandestine rebaptisée « diaspora criminelle » nigériane par Stephen Ellis dans This Present Darkness. L'auteur questionne, dans cette publication posthume, le rapport entre prospérité et univers spirituel dans le Nigeria d'aujourd'hui. Il affirme que pour bien comprendre le besoin pressant de s'enrichir qui concerne tous les secteurs de la société, il faut enquêter sur « certains éléments de la psychologie du crime organisé nigérian » (Ellis 2016 : 4), crime dont de nombreuses ramifications intéressent précisément l'émigration vers l'Europe. L'origine occulte de l'argent est véhiculée par certaines expressions entrées depuis longtemps dans le «marché linguistique », entre autres de la diaspora :

1. Le film As Above, So Below a été l'un des plus influents parmi ceux relevant de ce qu'on a appelé la «L.A. Rebellion Film Movement» ou bien la «Los Angeles School of Black Filmmakers ». Sorti en 1973, il a été réalisé par Larry Clark. Je reprends ici ce titre pour symboliser une certaine circularité de l'histoire ainsi que les imbrications entre violence, spiritualité et migration. Cette recherche a été conduite dans le cadre du Projet «Hégémonie, domination, résistance. Ethnographie des savoirs et des expériences de la subalternité » et du Projet « Mission ethnologique italienne en Afrique sub-saharienne » (à l'Université de Turin). 
depuis le "get-rich quick syndrome » jusqu'aux «Yahoo plus plus », et à l' "Ogun owo », c'est-à-dire l'argent rituel en passant par les «Mama-cash » et les «Mama-office» dont me parlent avec admiration les plus jeunes pour désigner ces femmes aisées disposant d'argent liquide et qui attendent, confortablement assises derrière leurs bureaux, tandis que leurs nouvelles maisons à étages sont bâties à toute vitesse à Benin City ou dans sa proche banlieue. Ce sont là les nouveaux vocables d'une migration théocratique ${ }^{2}$ nigériane $^{2}$ florissante et, dans le même temps, les images en chair et en os des nombreux multiplicateurs d'argent (money-doublers), qui évoluent apparemment avec aisance entre espace virtuel et espace rituel.

Ellis s'efforce d'élucider cette psychologie spirituelle de l'abondance, à laquelle tous veulent participer au Nigeria pour en retirer, tant bien que mal, un bénéfice personnel, aussi marginal qu'il puisse être au final. Toutefois, selon moi, l'auteur ne parvient pas à éviter les pièges d'un projet visant à questionner sur la longue durée des facteurs tels que le bien-être individuel, les inégalités sociales, la déviance et la spiritualité. En effet, lorsque dans ses conclusions il se confronte à la question "Why Nigeria? », il craint de sauter à pieds joints dans un culturalisme de bas étage. Après avoir analysé, page après page, l'histoire de l'Aruosa Church, du Long Juju, de l'autel d'Ayelala, et suggéré qu'on est, dans chaque cas, confronté à une « culture de la corruption » (Ellis 2016 : 223) qui, au Nigeria, a toujours eu « deux publics» (Ekeh 1975), il conclut : « Nigerian organised crime is not created by culture, but it does arise from a particular history » (Ellis $2016: 230$ ). Je ne pense pas que cette phrase éclaire mieux ou réponde mieux à sa question. Pourquoi donc le Nigéria ? Pourquoi cette crise sorcellaire constante, tenace, presque prévisible dans les vies de ces si nombreuses filles multiplicatrices d'argent?

De cette diaspora criminelle nigériane, je n'examinerai donc que celle qui organise le circuit des jeunes femmes sur les marchés européens de la prostitution, mais en prenant davantage à bras le corps certains faits « culturels » réfractaires au temps et qui, par certains aspects, constituent la

2. Dans Tribes of the Niger, TALBOT (1967) avait parlé d'«administration théocratique » à propos des régions du sud-est du Nigeria. J'essaierai de démontrer dans les pages qui suivent à quel point l'économie migratoire nigériane est gouvernée par la « volonté » des esprits et des divinités sur les autels desquels on prête serment, on contracte une dette et on promet de la rembourser. Il faut aussi rappeler à ce propos que le 9 mars 2018, l'Oba de Benin City Ewuare II, a déclaré pendant une séance rituelle publique sa lutte contre le pouvoir mystique de guérisseurs ("native-doctors »), lorsqu'il est utilisé pour nuire et exploiter les jeunes filles. Il a lancé une malédiction de mort contre n'importe qui mettra au service de la traite son pouvoir pour «blesser» dans la nuit et assujettir pendant le jour toutes ces «lucioles noires» (l'une des expressions italiennes pour nommer les filles nigérianes dans la rue). On mesure ici toute la proximité entre «administration théocratique » et «migration théocratique ». 
part d'inviolé de la conscience individuelle. Au vu de la forme particulière que prend la sorcellerie dans ces trajectoires migratoires, et que je décrirai dans les pages qui suivent, une théorie durable de la transformation de soi, des rapports sociaux et des objets de valeur se dessine.

On peut résumer de la sorte les antécédents de cette scène migratoire : la diaspora nigériane s'appuie sur un système particulier d'assujettissement, économique et religieux, qui gouverne la migration des femmes vers l'Europe et qui crée des disparités sociales et symboliques bien précises entre femmes jeunes et femmes adultes à partir d'un rite communément appelé « vaudou » et de la fabrication d'un objet-fétiche ${ }^{3}$. Je me concentrerai ici en particulier sur un lien persistant entre dette et sorcellerie qui influe sur les relations sociales entre femmes nigérianes immigrées et entre elles et les interlocuteurs italiens qu'elles rencontrent dans leur parcours d'intégration en Italie. D'un côté, la dette est celle que contractent les jeunes femmes devant un autel au début ou au cours du voyage, ou encore à leur arrivée dans un pays d'accueil. Son montant exact n'est toutefois pas précisé avant le départ et peut augmenter ou diminuer en fonction du nombre de passages, de main en main, auxquels est soumise la jeune femme dans son long « voyage de travail » (Aderinto $2015)^{4}$; nous verrons aussi que la dette est toujours ce quelque chose qui reste à payer à moins que ne soit fidèlement transcrit tout échange d'argent de l'une à l'autre (la liste des paiements varie à l'infini : depuis le loyer jusqu'à la somme à envoyer à la famille au Nigeria; du prix de l'emplacement où attendre les clients aux courses au supermarché ; de la coiffure à la mode à la quête de l'église le dimanche). D'un autre côté, la sorcellerie est l'horizon de sens qui permet d'appréhender les rapports de pouvoir et leurs conséquences directes. Les coups qu'elle porte sont toujours nocturnes et se manifestent par

3. Sur l'italò vudù (le vaudou italien), voir S. TALIANI (2016, 2017); sur la migration nigériane en France, voir le livre dirigé par B. LAVAUD-LEGENDRE (2013).

4. Dans cette économie charnelle se fait jour un type de femme marchandisable et contrôlable, comme c'était déjà le cas dans la migration transnationale coloniale en Afrique, dans ce trafic prolifique de «mines d'or errantes » qui a intéressé le Nigéria et le Ghana tout au long de la première moitié du XX $\mathrm{X}^{\mathrm{e}}$ siècle (NAANEN 1991). D'après L. FouRCHARD (2013), on peut tracer une généalogie historique des rapports sociaux basés sur le système du gage et de la dette qui gouvernait déjà dans l'Afrique orientale britannique le marché de jeunes et très jeunes femmes en danger moral et en même temps moralement dangereuses. Ces cadettes sociales allaient alimenter le «marché de viande fraîche » (GEORGE 2014), économie transfrontalière florissante à l'époque. On peut rapidement esquisser deux axes de continuité avec le cadre actuel de la traite de jeunes Nigérianes vers l'Europe à des fins sexuelles : le premier concerne la mobilité sociale et sexuelle encore aujourd'hui fortement territorialisée; le second est en rapport avec l'utilisation d'objets-fétiches et de rituels pour se protéger (de la maladie, de la violence du voyage, du mauvais sort) et/ ou attaquer (ceux qui sont insolvables dans le cadre du système). 
des visions, des rêves, des manducations mystiques, tandis que ses contrecoups sont diurnes, visibles aussi bien dans les atteintes physiques et les cicatrices sur les corps que dans les symptômes d'anxiété, d'angoisse, de résignation, d'agitation que ces jeunes femmes manifestent devant un médecin traitant qui s'empresse de les traduire dans le langage de la biomédecine et de la psychologie en stress post-traumatique, somatisation ou encore état psychotique aigu. Ayant déjà approfondi ailleurs ces aspects de la question (Taliani 2012), je ne m'y attarderai pas ici. Je me propose plutôt de questionner dans cet article deux notions, en m'appuyant d'une part sur les travaux de William Pietz (2005: 19), dont l'étude classique sur l'objet-fétiche porte sur « la sujétion du corps humain [...] à l'influence d'objets significatifs qui, bien que coupés du corps humain, fonctionnent à certains moments comme des organes de contrôle », et d'autre part sur les travaux de Patrice Yengo (2016) qui, récemment, évoque la figure du "père de derrière $»^{5}$ pour parler des mutations sorcières dans le bassin du Congo. Dans cette ethnographie de la crise sorcellaire dans la migration nigériane, aussi bien la notion d' " organes de contrôle» que la figure de la «mère de derrière » nous seront précieuses pour comprendre la «puissance noire nigériane» (Tonda $2015: 22$ ).

Mon travail se base sur une recherche ethnographique d'une vingtaine d'années, effectuée principalement en Italie, à Turin et à Naples ${ }^{6}$. J'ai rencontré des femmes nigérianes dans des communautés d'accueil, aux urgences ou encore dans des services psychiatriques hospitaliers; chez elles ou dans des structures de soutien psychologique, comme le Centre Frantz Fanon; et enfin dans les églises catholiques ou pentecôtistes qu'elles fréquentent le dimanche, dans des salles de tribunal où elles portent plainte ainsi que dans les services sociaux où elles reçoivent un premier accueil. Je n'analyserai aucun de ces lieux dans le détail, mon intention étant de me concentrer plutôt sur les moments de la «crise» : je prêterai attention à ces petits événements ordinaires - fréquents dans ce genre de migration — qui font irruption dans la vie des Nigérianes immigrées et qui provoquent une dérive liée à leur peur de se trouver confrontées à un destin qui a tout l'air de vouloir leur tomber dessus. Les signes de la déroute sont récurrents et presque toujours identiques : cessation du permis de séjour ou trop longue attente pour son obtention,

5. P. YENGO (2016) reconnait sa dette envers J.-P. WARNIER (1988) pour ce qui est de l'interprétation de la figure du « père de derrière »; il rappelle également l'article de J. BONHOMME (2005) sur le « voir par-derrière».

6. Les données rassemblent à la fois des extraits de dépositions dans des commissariats, des certificats rédigés par des membres du personnel hospitalier, des dossiers sociaux et juridiques (la plupart m'ont été fournis par les femmes au cours de nos entretiens) ainsi que des extraits d'entretiens et de conversations que j'ai pu avoir ces dernières années (1997-2017). 
expulsion du logement ou licenciement (mais, encore plus, impossibilité d'obtenir un contrat ne serait-ce que de quelques heures), hospitalisation, diagnostics médicaux, nouvelles de deuil ou de maladie qui ne sont pas totalement inattendues... En raison de l'importance accordée au thème de la crise, je m'efforcerai de dialoguer dans ces pages avec la critique récemment adressée à la crise sorcellaire dans les études anthropologiques (Warnier 2017).

L'article comprend deux parties ethnographiques, une pause de réflexion méthodologique et une conclusion. Je commencerai par mettre en place l'ossature des rapports de dépendance et d' " obéissance superstitieuse » (Bernault 2009) qui doivent être maintenus dans les contextes actuels de la diaspora pour qu'il puisse y avoir production de richesse. Je raconterai ensuite l'histoire de ma rencontre avec Hannah et avec sa « crise ». Plusieurs questions méthodologiques fondamentales seront soulevées au sujet de la réalité des pouvoirs magiques et du rôle du chercheur de terrain. Enfin, la conclusion propose une hypothèse de lecture de cette psychologie de l'abondance qui est en même temps une théorie anthropologique du temps à venir.

\section{Une ethnographie des sorts migratoires}

Chacune des histoires migratoires dont les femmes nigérianes sont actrices commence par un acte rituel destiné à naturaliser une différence sociale arbitraire (selon laquelle les aînés contrôlent et gèrent littéralement la vie des plus jeunes) et à créer des relations de pouvoir asymétriques (dans lesquelles plus la femme adulte se fait autoritaire et agressive, plus la jeune fille se soumet et exécute les ordres). Le pouvoir du rite est tel qu'il parvient même à inverser l'ordre des générations, puisqu'il fait toujours de la «madame ${ }^{7}$ une « aînée », même si celle-ci peut être plus jeune que les femmes qu'elle amène. De fait, le terme $e d o^{8}$ désignant la «madame » qui exploite signifie littéralement « mère des fesses » ou « mère de derrière » (iye onisan) : celle qui a tout pouvoir sur la vie de ses « filles » (obviye) ${ }^{9}$. Obviye (ou oviye) désigne

7. «Madame » est l'expression la plus utilisée dans le langage pidgin (et désormais en italien aussi) pour designer l'aînée des femmes, l'exploiteuse.

8. Tous les termes que je reporte ici — comme d'autres dans le corps du texte — sont en langue edo, parce que la majorité des femmes rencontrées proviennent de l'État d'Edo (ou $\grave{E} d o ́$ ), dans le sud-est du pays, et parlent la langue edo (ou bini), même si elles ne sont pas toutes nécessairement originaires de Benin City et si, dans quelques cas, elles parlent d'autres langues telles que l'urhobo, l'ishan, l'esan, etc. Pour la transcription des mots utilisés dans la région, je préciserai les différentes options adoptées par les auteurs.

9. Dans sa recherche sur l'organisation sociale publiée en 1957, BRADBURY (2017: 27) fait une distinction entre les termes ovi erha (l'enfant du père) et oviye (l'enfant de la mère). AgHeYsi (1986) traduit le terme "obvi » par " child of » (et, donc, associé dans ce cas 
littéralement «l'enfant du côté de la mère " (que l'on traduit en anglais aussi bien par "mother's child» que par "child of one's mother »), à savoir les frères et sours nés de la même mère; tandis que iye onisan est une expression de dénigrement: si iye désigne la "mère», onisan indique vulgairement le «cul» (ou mieux l'« anus », comme dans certain proverbes).

Le lexique de la parenté et celui de la compensation, typique de l'alliance, ne cessent ici de s'entremêler en un ensemble indifférencié. Ce qui s'instaure, en même temps que la dette économique vis-à-vis d'une personne et que la dette symbolique face à une divinité ou à un esprit, c'est une hiérarchie précise dans laquelle les cadettes sont toutes endettées auprès d'une seule et unique mère de derrière. D'où une relation d'appartenance-dépendance, qu'exprime bien le terme edo " ese » (ou " èsé ») (littéralement « faveur», « don», «bon »), ou une forme particulière de remerciement et de gratitude ${ }^{10}$.

Au cours du rite, on jure sur l'autel de la divinité choisie de s'engager à respecter l'aînée, à rendre la somme pour laquelle on vient de s'endetter, à respecter le pacte sous peine de subir toutes sortes de calamités (dans un crescendo de maladie, de folie, de mort). Ce genre de rite peut être pratiqué aussi bien au Nigéria, avant le départ, qu'à l'arrivée, dans un pays de transit ou dans le pays d'accueil (dans les toilettes d'une gare, au bord d'une rivière ou plus commodément dans un appartement loué à cet effet). Dans les deux cas, on vise à faire en sorte que le sujet «épidermise » puissamment la hiérarchie des générations, en soumettant le cadet à l'aîné. J'utilise ici le mot « épidermiser », dans un sens double. D'une part, j'entends évoquer le fait que cette hiérarchie est incorporée à coups d'entailles, de lésions, de prélèvements de certaines parties du corps : des bouts de peau sautent, des morceaux d'ongles partent, des touffes de cheveux et des poils pubiens sont recueillis en même temps que des caillots de sang menstruel. Il s'agit donc d'une vie troublée par des objets-fétiches qui sont des parties de leurs corps (ses morceaux détachables et détachés au cours du rite). De l'autre, ce terme rappelle que les punitions, les brimades, les contraintes qui s'abattent sur les corps de ces jeunes femmes (si elles se rebellent, si elles brûlent de se racheter ou de s'émanciper, si elles ne ramènent pas assez d'argent ou si elles refusent de travailler) peuvent être d'une férocité extrême : ciseaux, couteaux, brûlures, poinçons et fers

à iye, qui veut dire «mère », ce qui permet de comprendre la traduction ci-dessus). En analysant les termes employés, on voit bien de quelle manière s'élabore une forme de « parentèle fictive » entre la jeune femme amenée en Italie et sa madame et comment, dans le même temps, s'instaure une relation de descendance/dépendance fortement asymétrique basée sur le vocabulaire à la fois de la descendance/filiation et de l'alliance, à partir d'une transaction économique et du passage entre dot-gage-dette.

10. Transcrit « èse » par MELZIAN (1937:56) le terme est traduit en anglais comme " goodness » et «favour». 
à repasser servent à infliger de la douleur. Des surfaces du corps ou du cuir chevelu attendent ainsi une longue épidermisation, après des interventions chirurgicales plus ou moins invasives : restent des épaississements de la peau et des cicatrices pour ne pas oublier.

Or, même si les femmes étaient parfaitement consentantes au moment de sa fabrication, l'objet-fétiche (Ebo ou èbò $)^{11}$ provoque en elles une véritable terreur lorsqu'elles comprennent sa fonction, son objectif, sa durée et sa violence : autant de variables désormais indépendantes de l'intention originelle, et qui vont bien au-delà de leur volonté ou de leur désir. En prenant vie de manière autonome, l'objet fabriqué prend à son tour vie en elles, dans un vertige d'angoisses, de cauchemars, de fantômes, dont on ne sait à qui parler. Lorsque j'arrive à leur faire partager ces peurs, c'est toujours en l'absence de la chose - car l'objet-fétiche est ce qui reste dans les mains de quelqu'un d'autre - et le récit prend des directions imprévues : j'écoute un foisonnement de souvenirs dans lesquels d'autres épisodes, de nouveaux événements ou des faits inexplicables ont entretenu l'inquiétude, essentiellement parce qu'on ne savait pas quoi penser. Parfois, le rapport entre rite, objet-fétiche et peur est direct : auparavant, pendant le rite, on a subi des scarifications sur la poitrine pour recueillir des gouttes de sang ou on a été menacé par des flammes; et puis, après de longs mois, voire des années, on se plaint de brûlures sur le sternum ou de chaleur excessive dans la poitrine. D'autres fois, il prend la forme d'un lien métaphorique, en déplaçant sur l'image onirique la scène de la manducation rituelle : la personne ne peut s'empêcher de cracher régulièrement, dans un petit récipient en plastique qu'elle est désormais obligée d'avoir sur elle, la

11. Voir pour la définition du terme obo (pluriel $e b o$ ) ce qu'en dit BRADBURY $(2017: 59$, notre traduction): «Tous ceux qui pratiquent la magie peuvent être réunis dans la catégorie d'obo [...], terme généralement traduit par "médecin". » Sachant que le savoir médical est souvent conservé et transmis au sein de la famille, le terme ebo était réservé «à ceux qu'on considérait comme particulièrement compétents en la matière », experts en « soin, divination, lutte contre les sorcières, supplices » (ibid.). Le pluriel d'obo est donc ebo, forme que les jeunes femmes emploient généralement pour désigner le « fétiche » fabriqué au cours du rituel. AHIABA (2015) nous rappelle qu' Ebo était l'esprit et que ode était l'idole ou le fétiche, mais aujourd'hui cette différence a disparu dans le langage courant. L'auteur ajoute que la mutation observée est sans doute en rapport avec la traduction proposée par les missionnaires chrétiens qui avaient traduit par « fétiche» le mot «Ebo ». En effet, dans d'autres travaux plus anciens, "ebo » apparaît comme synonyme des « esprits» cohabitant avec les ancêtres et autres divinités du panthéon, à côté de Osa, Ogun, Olokun, etc. (Northcote 2010 [1910]). Selon Melzian (1937: 47), èbo était « any charm of powdery substance with which peolple wash themselves or rub their foreheads or chests. [...] Mostly of noxious character, but also for preventing diseases and bad luck ». Il avait déjà la même connotation que le terme pidgin «juju » (ibid. : 160). En yoruba, le terme signifie « sacrifice »; il est aussi utilisé aux Caraïbes et aux États-Unis chez les immigrés caribéens adeptes du culte cubain de Lukumí Orisha (NodAL \& Ramos 2005). 
salive qu'elle secrète suite à une gêne à la bouche ou à la gorge après avoir mangé et bu « la nuit »" ${ }^{12}$. Dans un autre cas encore, Monica me raconte pour la première fois ce qu'elle définit comme une «scène d'horreur » qu'elle ne sait — me dit-elle — à qui d'autre raconter :

S'étant rendue à la cuisine pour préparer à manger et ayant mangé, elle laisse la casserole couverte à refroidir hors du frigo ; lorsqu'elle revient moins d'une demi-heure plus tard pour la mettre au réfrigérateur, elle soulève le couvercle et y trouve des dizaines et des dizaines de fourmis. Alarmée, elle essaie de comprendre d'où elles viennent mais elle ne voit aucune «file» à l'extérieur de la casserole pouvant indiquer une voie d'accès. Terrorisée, elle jette le tout (entretien avec M. N. O., 13 juin 2017, Naples).

On ne peut pas imposer aux expériences une signification univoque, ni une interprétation dominante ayant exclusivement sa raison d'être dans le rite fait avant d'émigrer. Cependant il me semble qu'à partir de ces scènes d'horreur et de ce cri («scream », dit-elle), on peut bien admettre que la peur liée à l'efficacité du rite se révèle comme quelque chose de plus obscur, comme le retour d'un passé vivant et menaçant. Pour Monica, par exemple, les fourmis renvoient à des souvenirs terrifiants de son adolescence lorsqu'au collège elle avait vu un jour dans la cour de l'établissement scolaire l'une de ses maîtresses parler avec un arbre auquel on attribuait des pouvoirs magiques; ou quand le fondateur de l'école, décédé depuis longtemps, s'est présenté devant elle, dans sa chambre, à la manière d'un fantôme. Elle a commencé alors à expérimenter des visions, qui même aujourd'hui ne la laissent pas tranquille et qui avaient motivé, à l'époque, l'abandon de ses études et de son projet d'émigrer en Europe. Dans son cas, je n'ai pas pu recueillir jusqu'à présent d'éléments qui pourraient renvoyer directement l'épisode décrit plus haut à l'expérience vécue pendant le rite, mais il renvoie immédiatement à sa migration et à l'angoisse qui accompagne la conscience d'avoir une dette à régler. On peut donc considérer ici l'objet-fétiche comme ce qui, par son statut et son lien avec l'individu, expose ce dernier à de graves risques psychiques. Cette définition contredit celle de la psychanalyse, qui voit plutôt dans le fétiche un objet capable de préserver le sujet d'une angoisse de castration qui n'est tolérable qu'au prix d'un compromis extrêmement coûteux. Ce que ces jeunes femmes redoutent par dessus tout, c'est une souffrance, une maladie ou une mort qui ne pourraient plus être considérées comme accidentelles.

Le rite fabrique donc un type de femme cadette, en affirmant devant Dieu des principes d'inégalité au sein des relations sociales. Le corps de celle-ci

12. Je ne peux pas reprendre ici en détail le cas de Georgia et Tina, décrits dans un récent travail dédié au fétiche dans la migration nigériane (TALIANI 2016). 
est alors transformé en un « corps-fétiche », selon une logique opposée à celle décrite par Luc de Heusch (2000) à propos de la nature du corps du roi de Kongo. C'est dans ce contexte qu'a d'abord été forgée l'expression de corps-fétiche, «pour expliquer la possibilité d'une action sur la nature d'un corps singulier transformé par une opération rituelle - celle-ci étant à la fois une initiation et une intronisation - qui lui confère une surpuissance » (Adler 2002 : 174). Dans le cas des immigrées nigérianes, ces rapports changent de sens et il s'agit plutôt d'une opération rituelle transformant un individu singulier en un corps-fétiche qui ne sera jamais en mesure d'exercer un pouvoir. Qu'il s'agisse de violence symbolique ou de coercition physique sous toutes ses formes, c'est bien l'adulte qui est à l'origine des innombrables brutalités infligées aux plus jeunes, entre virulence et rancœur. L'objectif du rite est de renforcer une norme sociale largement partagée et répandue dans le contexte socio-pédagogique nigérian, en la faisant apparaître comme « naturelle », autrement dit en la dé-historicisant. En ce sens, le rite est plus efficient qu'efficace. J'entends par là que nous ne pouvons pas l'interpréter dans une perspective de résolution de la crise, donc en termes d'efficacité symbolique : dénué de fonction thérapeutique, abréactive ou cathartique (contrairement à ce qui se passe en contexte de cure), le rite vise ici uniquement à soumettre des sujets en quête de subjectivation.

Cette économie charnelle affirme donc des principes d'inégalité devant différents dieux. On a ainsi affaire à une parodie rituelle sanglante ${ }^{13}$, qui s'appuie sur un processus de subjectivation de l'initiée, sur son désir d'autonomie financière et d'indépendance d'avec les logiques familiales, pour pouvoir mieux l'assujettir à une forme de domination fondée sur un écart générationnel factice et sur une relation d'endettement. Ce processus va de pair avec une accumulation de capital extérieur à l'économie domestique et locale. Reste à comprendre pourquoi le rite est pratiqué aussi systématiquement tout au long du parcours migratoire, avant le départ, mais aussi, de plus en plus souvent, au cours du voyage et après l'arrivée en Europe.

Les mutations internes et externes engendrées par la migration sont si radicales que le rite est utilisé par le groupe pour fixer l'individu le plus longtemps possible aux valeurs, normes et pratiques de son système culturel et social, pour prévenir, ou plutôt pour différer un changement que l'on sait pourtant inéluctable. S'il est vrai que le rite est ce qui permet de mettre en scène ce qu'on

13. Je reprends ici, en la modifiant, une expression proposée par D. PRATTEN (2007) dans son analyse de la manipulation des serments rituels parfois mise en œuvre à l'époque coloniale au Nigeria devant certains autels par des notables et fonctionnaires locaux. Ceux-ci entremêlaient, au cours des rites, traditions locales et normes coloniales pour en tirer un maximum de profit dans le sens de leurs intérêts. Prêter serment, dans ces cas-là, devenait selon PrATTEN (2007: 88) une véritable «parodie des pratiques traditionnelles ». 
ne peut dire autrement, en l'occurrence les paradoxes de la migration et les transformations auxquelles elle soumet les individus, alors cette caractéristique demeure ici intacte. Il ressort en effet du récit de ces jeunes femmes que celles qui se prostituaient déjà au Nigeria n'ont jamais été soumises à un rite quelconque avant de partir pour l'Europe ${ }^{14}$ : il y a donc bien un lien particulier qui unit le rite « vaudou » et la migration. En outre, si aucune mère de derrière ne s'attend vraiment à recevoir la somme fixée au moment du pacte, elle cherche en revanche à maximiser le profit, s'il le faut par la violence, tant que la cadette est sous l'emprise de la panique. Car elle sait bien que celle-ci finira tôt ou tard par se libérer de ce sentiment et du pacte lui-même, et ce dès que lui sera révélé le caractère historique de la domination qui s'exerce sur elle.

La migration est donc une expérience qui expose à l'historicisation des institutions et des normes et qui, partant, dénaturalise ce que le rite avait tenté de dé-historiciser, en un cycle de révélations continues où s'alternent l'enthousiasme et l'incertitude. Tout se passe comme si c'était précisément parce que la migration met en jeu une frontière purement matérielle - un espace concret à traverser - que la décision même de le franchir active nécessairement une série de rites destinés à faire incorporer ce qui n'est pourtant qu'un secret de polichinelle. Car tout le monde connaît le désir de rachat de ces femmes, désir d'accéder à des anthropologies alternatives où les rapports hommesfemmes, familiaux et générationnels sont pensés et construits autrement. Et c'est précisément pour cette raison que les mères de derrière tenteront par tous les moyens d'exploiter la panique morale qui accompagne l'isolement propre à l'expérience migratoire, tant que ses filles manquent de réseaux et de contacts : il faut que tous ceux qui participent à cette économie occulte (y compris les familles) puissent y trouver leur compte. Dans ce processus lent, le rite ne vise qu'à contrôler le moment de la rupture et du détachement. S'il perd trop rapidement de son efficience, il sera donc réitéré en Italie (ou ailleurs dans le pays d'accueil) pour maximiser le profit en prolongeant ses effets déstabilisants sur l'organisation psychique de la jeune fille. C'est précisément parce qu'il ne cesse d'échouer, que le rite doit être régulièrement répété. Prenons un exemple de ces trajectoires trafiquées du rite. Il s'agit de l'extrait d'un acte juridique destiné à la défense d'une Nigériane dénoncée par une femme plus jeune pour trafic et exploitation (nous sommes en GrandeBretagne) $)^{15}$. Dans le document juridique, les termes sont traduits de l'edo ou

14. J'ai recueilli ces informations au cours d'une recherche de terrain menée en décembre 2010 et janvier 2011 à Bamako, au Mali, dans le quartier Bla-bla où se trouvaient, outre de très nombreuses jeunes filles maliennes, des prostituées nigérianes et ghanéennes.

15. J'ai reçu par courriel en 2015 une demande d'expertise de la part de l'avocat de l'accusée, qui souhaitait avoir l'avis d'un expert anthropologue. 
du pidgin anglais en anglais (on peut aussi imaginer que les femmes se sont directement adressées aux juges et aux avocats en anglais). "Iye onisan » devient « Mama », « e bo », " juju », et les différentes sphères spirituelles sont évoquées dans le vocabulaire passe-partout du magico-religieux ${ }^{16}$ :

Ms Augusta [pseudonym] is due to appear before the Central Criminal Court on $30^{\text {th }}$ July 2015 for a Plea and Case Management Hearing ( $P C M H »)$ in relation to an allegation, inter alia, that she trafficked one Ms Jane [pseudonym] into the United Kingdom for the purpose of sexual exploitation [...].

The allegations that Ms Augusta now faces follow in the wake of a trial at Harrow Crown Court that involved similar allegations. At the conclusion of that trial, Ms Augusta was acquitted of all of the 17 counts that she then faced. Ms Augusta's defence in both cases is the same. She asserts that she has been set up by the mother of one of the complainants in the Harrow matter, whom she knew as «Mama». Ms Augusta asserts that she was first trafficked to Italy by Mama, and having been deported from there to Togo, was then trafficked into the United Kingdom. Thereafter, she asserts that, because she refused to continue paying Mama, Mama has taken revenge by setting her up.

Prior to being trafficked into the United Kingdom, Ms Augusta asserts that she participated in a « juju» magic ceremony during the course of which she was ritually marked, causing scarring. Ms Jane also asserts that juju magic was employed to control her. However, she does not allege that she was subject to any ritual before travelling from Nigeria, instead she gave a (somewhat vague and incomprehensible) account of Ms Augusta performing some kind of juju ritual in her home in the United Kingdom (P.E., dossier juridique, mémoire de l'avocat qui la défend, 2015).

Dans le document, le juju est évoqué par l'avocat, qui rapporte « fidèlement » ce qui a été déclaré aussi bien par la victime présumée que par la maquerelle supposée, pour raconter une agression subie dans le cadre de l'exploitation de la femme sur la femme. Les voix se confondent, l'identité de l'agresseur ne semble plus si facilement reconnaissable et il est certain que le contexte juridique génère une stratégie défensive bien précise, visant à construire, et pas seulement à restituer, un discours autour du fétiche. Comme dans d'autres ethnographies dont les domaines de recherche ont été des cours

16. Pour pouvoir avancer et pour proposer une ethnographie affranchie des obsessions classificatoires, des compartimentages et des stagnations disciplinaires, peut-être serait-il bon de s'inspirer de la suggestion de J.-F. BAYART (2014: 47) quand il propose tout récemment de (ré)adopter une «nomadologie » à la Deleuze et Guattari : «Une nomadologie qui ne raisonne plus dans les termes stratégiques de l'individualisme méthodologique, ou holistes du culturalisme, ou binaires de la lutte des classes, mais dans ceux de la "multiplicité" des "agencements" producteurs de l'historicité. » Voir DeleuZe \& GuatTARI (1980). 
de justice et des tribunaux ${ }^{17}$, on comprend bien à quel point les objectifs processuels produisent un objet de recherche qui n'appartient pas plus aux femmes nigérianes qu'il n'est du ressort des avocats (anglais, italiens ou africains) : dans ce cas précis, le cabinet légal voulait approfondir ce que le savoir anthropologique pouvait dire d'un rite juju «fait maison» au cours duquel était fabriqué un objet « bricolé » (dans le mémoire légal, l'objet-fétiche devient un " objet-diy », à savoir " do it yourself»). Augusta, la défenderesse, considérait en effet qu'elle avait fait l'objet d'une vengeance de la part de la mère de son accusatrice, connue seulement sous l'appellatif assez vague de «Mama », utilisé couramment pour désigner n'importe quelle femme d'âge mûr. Elle affirmait, pour sa défense, qu'un rite juju, lorsqu'il n'est pas pratiqué en présence d'un autel et d'un guérisseur, n'a pas grande crédibilité aux yeux d'une « victime» potentielle, car sa volonté spirituelle est faible.

Certaines jeunes Nigérianes avec qui j'ai parlé au fil des ans ajoutent au mot « $e b o$ » le terme «blanc», renvoyant par là à sa faiblesse plutôt qu'à sa couleur. Car le fétiche, s'il est toujours noir (en tout cas d'une couleur sombre et difficilement définissable), peut être plus ou moins «fort» ou « léger », c'est-à-dire blanc, selon la force ou la faiblesse de l'esprit incarné dans l'objet.

«Je n'y crois pas, mais ça existe»

Joseph Tonda (2015) reproche aux professionnels et administrateurs blancs, ainsi qu'aux anthropologues et sociologues de l'Afrique, de tomber dans le panneau de l'éblouissement noir. Récemment, dans L'impérialisme postcolonial, il critique sans hésiter toutes ces sociétés, aussi bien africaines qu'européennes ou américaines, qui se sont laissées séduire par l'invisible noir, par l'objet noir, par la chose noire et qui ont attribué une valeur inestimable au pouvoir mystique, spirituel et magique noir (comme c'est arrivé aux avocats et aux juges dans le cas d'Augusta évoqué ci-dessus).

La critique de Tonda, qui ne saurait être plus tranchante aussi contre le savoir des sciences sociales, m'interpelle sur la façon de parler de mes rencontres avec le « complexe sorcellaire » (Warnier 2017). Peut-être colonisée moi-même par l' ' intangible primitif» (Tonda 2015) de mon imaginaire de chercheuse, finirai-je par n'être qu'un « agent de fonction » parmi d'autres de l'impérialisme postcolonial lorsque je parle avec ces femmes de la sorcellerie

17. Il est impossible de citer toute la littérature sur ce sujet, dont l'imposante publication dirigée par É. DE ROSNY (2006), Justice et sorcellerie. Je me limiterai ici à rappeler les travaux portant sur le contexte européen de la Cour pénale internationale : ClAVERIE (2015), DEZALAY (2017). 
qui les attaque ? Il me semble que Warnier lui aussi, lorsqu'il évoque «l'effet Magritte de la sorcellerie $»^{18}$, quoique sur des bases différentes, avance prudemment dans la même direction. Pourtant — c'est là ma question —, ne pouvons-nous pas en tant qu'anthropologues, ne pas jeter le bébé avec l'eau du bain? En nous reconnaissant comme agents de fonction de la sorcellerie, ne pouvons-nous voir une quelconque forme d'autodétermination dans le « dire et agir» magico-sorcellaire et fataliste en expansion sans déboucher sur un assourdissant éblouissement ou encore un trompe-l'œil ?

Avant de tenter de répondre à cette question, j'évoquerai mes deux entretiens avec une jeune Nigériane que j'appellerai Hannah ${ }^{19}$, pour décrire ma rencontre avec la « chose noire» dont parle J. Tonda.

Hannah est une belle jeune fille de vingt-cinq ans, qui a porté plainte contre une compatriote plus âgée. À partir de ce moment-là, elle a été incluse dans un programme de réinsertion. Elle s'est prostituée pendant deux ans, dans une riche province du nord de l'Italie, pour payer la dette contractée au Nigeria (dette qui est «passée» de madame à madame et qui s'élevait à environ 45000 euros). Une fois une partie de la dette payée, elle a continué à se prostituer pendant plusieurs mois, en gardant pour elle-même et sa famille une bonne partie de ses gains, mais aussi en continuant à payer le prix du join (l'emploi) à d'autres femmes nigérianes. Un jour de novembre, après de nombreuses disputes et des coups reçus pour avoir interrompu le paiement du

18. L'auteur écrit que l'effet Magritte de la sorcellerie est « le fait de prendre [...] le tableau de la pipe pour la pipe, le récit pour la chose, la rumeur pour la crise avérée » (WARNIER 2017 : 130). Il évoque donc une certaine confusion entre représentation et substance, et un surinvestissement des interprétations sorcellaires en Afrique aujourd'hui. D'où le titre provocateur de son travail, Ceci n'est pas un sorcier.

19. Toutes les citations dans ce texte proviennent des entretiens avec «Hannah», F.I., avril 2010, à Turin. Ces rencontres ont eu lieu au Centre Frantz Fanon où Hannah s'est présentée avec une éducatrice de sa communauté d'accueil pour parler, à sa demande, avec quelqu'un qui connaîtrait sa «culture », mieux que le médecin qui lui avait rendu visite quelques semaines auparavant. Il n'est pas dans mes intentions de reconstituer ici le débat féroce autour des laboratoires d'ethnopsychiatrie, surtout en France. Je tiens simplement à souligner que, comme le signalaient déjà M. C. \& E. OrTigues $(1984: 17)$ au sujet de l'expérience pionnière de Dakar-Fann : « On ne peut concevoir utilement la collaboration entre ethnologue et psychanalyste si l'on ne perçoit clairement au départ non pas seulement ce qui les distingue mais ce qui les oppose. \ Or, ce qui les oppose est, selon les auteurs, le rapport au savoir et surtout au questionnement : dans la recherche, c'est l'ethnographe qui formule la demande d'informations auprès de son interlocuteur en préconisant la voie à suivre afin de s'informer et d'instruire son étude, alors que dans la consultation auprès du psychanalyste, la demande doit toujours émaner du sujet, le savoir éventuellement acquis lui étant destiné ( $C$ 'est l'interrogation du sujet qui oriente et structure la situation » [ibid.]). Je propose ici de considérer la sorcellerie comme une situation tout à fait inédite parce qu'elle interroge le sujet et le chercheur en même temps dans un contexte donné. 
join, Hannah se rebelle et, à son tour, menace. La dernière madame, une fille dont elle était amie (parce que dans un premier temps elles avaient été obligées toutes les deux de se prostituer pour le compte d'une troisième Nigériane) l'agresse alors avec une lame de rasoir et lui fait une profonde entaille au visage. La cicatrice, qui souligne son œil gauche de part en part, s'élargit vers le bas du visage, descendant en forme de goutte vers la joue.

La police intervient à la suite d'un appel téléphonique qui signale la bagarre et, à la suite de la plainte présentée par Hannah, arrête la madame. Hannah est soignée puis transférée dans une autre ville du Nord, insérée dans un programme de protection.

Au cours de notre première rencontre, Hannah parle des symptômes qui l'affligent. Tandis qu'elle raconte certains cauchemars - deux femmes noires la touchent, lèvent la main sur elle (elle en imite le geste) - elle éclate en sanglots. Elle passe de l'italien à l'anglais. Puis elle s'arrête et s'excuse, car elle sait que son éducatrice ne comprend pas sa langue. Je lui assure que je traduirai pour elle, si elle veut, et qu'elle pourra donc continuer à parler en anglais. Dans un flot de paroles, Hannah déclare qu'elle a été empoisonnée dans la nuit: "You know, such spiritual things. » Puis, calmement, elle continue en décrivant comment, après avoir été à l'hôpital, et après que les médecins lui ont déclaré qu'elle était en excellente santé, au lieu de se tranquilliser, Hannah éprouve une sensation pernicieuse de peur, de panique : c'est ce qui se passe chez la plupart de ces femmes qui, en l'absence d'un diagnostic biomédical, sont d'autant plus saisies d'une angoisse de mort profonde ${ }^{20}$.

"I called my mother », elle regarde l'éducatrice et baisse les yeux, "Sorry. I did not tell you that ». Elle dit à sa mère que quelque chose la dérange et que les médecins blancs n'arrivent pas à voir ce que c'est. Elle baisse de nouveau les yeux, puis elle poursuit. Sa mère, après avoir consulté deux pasteurs de deux églises différentes (l'une, pentecôtiste, l'autre, évangélique), lui communique leur réponse à tous les deux : «empoisonnement». On comprend sa honte lorsqu'elle parle de tout cela. Hannah a peur du jugement de son éducatrice. Elle répète : "Moi, je ne crois pas à ces choses-là. Eux [elle se réfère aux éducateurs] savent que je suis une fille intelligente, je n'y crois pas. »

M'appuyant sur ce qu'elle laisse entrevoir au sujet de ses croyances et ce qu'en «savent » les éducateurs, j'insiste pour mieux comprendre son propre

20. La sorcellerie est un système de pensée qui se présente très souvent comme «postrationnel », car il intervient lorsque la raison n'est pas parvenue à apporter les réponses escomptées. WARNIER (2017 : 133) reprend ce point : « Le diagnostic d'attaque en sorcellerie n'est que très rarement formulé en première instance. Il l'est en dernier recours, tant il est lourd de menaces et d'angoisse. Quand les circonstances s'y prêtent, il est précédé d'un itinéraire thérapeutique dans lequel la biomédecine ou une investigation pragmatique des erreurs commises et des causes de l'échec figure en première intention. » 
rapport avec ce qu'elle croit, concernant « ces choses-là », le Mal et la maladie, dans la matérialité de la croyance (dans les actions bien avant que dans ses représentations, d'après de Certeau [1981]).

Moi : Dans ton rêve, est-ce que tu as mangé quelque chose ? Ces femmes t'ont donné à manger?

Elle ne se souvient pas de ce qu'elle a mangé, mais elle est sûre d'avoir mangé, répond-elle. Moi : Est-ce que tu sais si ta mère est allée aussi chez un native-doctor?

Elle : Non ma mère est une femme simple. Elle ne va pas chez les native-doctors. Je ne le lui permettrais pas.

L'observation sur le fait que sa mère est une femme simple me rappelle des propos déjà entendus ailleurs — au Cameroun, pendant ma recherche autour de l'evu et de la fabrication des enfants bulu - concernant le monde invisible de la sorcellerie où l'on distingue entre personnes innocentes et doubles, simples et mystiques.

Moi : Tu n'es jamais allée chez un native-doctor?

Elle : Non [silence]. Oui. Quand j'étais petite, ma mère m'y a menée quelquefois. Et puis, avant le voyage, on m'a amenée chez un native-doctor avant de partir pour l'Italie. Mais je ne dois pas parler de ces choses, on m'a dit que je ne dois en parler à personne.

Au cours de la deuxième rencontre, après un moment initial de repositionnement réciproque (nous parlons d'école : elle vient de passer les examens du Brevet; de la vie communautaire : elle va peut-être commencer un emploi saisonnier; de la famille : je lui demande comment vont les siens), nous reprenons là où nous nous étions arrêtées.

Moi : Devant quel autel as-tu juré avant de venir en Italie?

Elle : Je ne connais pas les autels.

Moi : Tu ne connais pas non plus Shango, Ogun, Olokun...?

[Elle rit. Elle porte sa main à sa bouche et son corps se penche en avant.]

Elle : Tu connais ces noms ? [Puis elle redevient sérieuse.] C'était sûrement un autel, mais je ne sais pas de quelle divinité. Je ne crois pas aux juju. Je ne me suis pas souciée de savoir de quel autel il s'agissait, je ne veux pas avoir d'autel chez moi, c'est éprouvant d'avoir un autel chez soi.

Je m'aperçois que son discours mêle des plans différents : de l'autel du native-doctor, elle passe à un autel domestique. Le dialogue s'articule - parfois sur un ton critique et polémique de la part d'Hannah - autour des représentants, matériels et symboliques, de son expérience migratoire, 
une expérience marquée par les règles de la traite et de l'obligation à la prostitution pour rembourser la dette qu'elle a contractée. À un moment donné, la figure d'Olokun devient un point central du discours, car la mère de sa première madame lui a dit un jour au téléphone : «Tu es fille d'Olokun, ne l'oublie pas.»

Juste avant, en parlant de sa première madame, Hannah avait dit que cette femme «avait peur » d'elle («Elle ne m'a jamais frappée, ni menacée. Elle avait peur de moi »). Hannah pense que sa mère, après avoir elle-même parlé avec la mère de la madame, voudrait qu'elle fasse quelque chose. De son côté, elle a dit à sa mère de ne pas continuer avec « ces choses », qu'elle-même ne fera absolument rien, qu'elle n'y croit pas. Pour ma part, j'interviens parfois dans ses propos en plaisantant; d'autres fois, mon ton se fait sérieux. Nous rions et nous nous taisons. Je la laisse répéter plusieurs fois qu'elle « ne croit pas » même si, selon moi, nous sommes déjà pleinement dans le registre de ces croyances. J'essaye de la faire osciller entre les deux façons (que je connais) de parler des croyances, sur un ton tantôt sérieux, tantôt badin.

Moi : Alors, tu vas me dire que tu n'as jamais vu Mami Wata au marché ou à la plage?

Elle : Non!

Moi : Même pas à Onitsha ?21.

Elle, en souriant : On dit qu'on peut la voir à Onitsha.

À la fin de notre rencontre, Hannah me demande si elle peut me poser une question. Je réponds que oui.

Elle : Toi, qu'est-ce que tu sais des native-doctors? Tu as déjà travaillé avec eux ? Qu'est-ce que tu en penses?

Moi : Il m'arrive, quand je suis en Afrique, de travailler avec certains guérisseurs. Ce que je peux te dire, c'est que j'ai rencontré des charlatans, que j'en ai rencontré d'autres sérieux et honnêtes. Je voudrais seulement savoir ce qui te trouble, ce qui rend ta vie pénible actuellement. Je n'exclus pas que ce qui s'est passé, ou d'autres choses encore dont nous n'avons pas eu le temps de parler pendant le peu de temps que nous nous sommes vues, puisse te troubler ou t'agiter, puisse te faire du mal. Parfois, pour expliquer la douleur que nous éprouvons en nous, une seule réponse ne suffit pas. Plusieurs expériences combinées pourraient nous avoir fait du mal. J'ai rencontré des guérisseurs qui étaient en mesure de pénétrer les replis de la souffrance d'hommes et de femmes, d'enfants et de vieux, pour faire disparaître et soigner une à une les plaies et les cicatrices que la douleur avait laissées. Tous ne sont pas comme ça. Mais si ta mère ou toi... vous vous étiez fiées à

21. Hannah est originaire d'un village situé dans l'est de l'Edo State. Elle parle l'ishan. Elle m'avait raconté au cours de notre premier entretien qu'elle se rendait avec sa mère 
quelqu'un au Nigéria, si tu t'y fies encore, j'écouterais avec attention ce que tu as à me dire et ce qu'eux ont dit.

Elle : Mon grand-père, le père de ma mère, était un native-doctor, un des plus importants de ma région.

À en croire...

Dans les échanges entre Hannah et moi, il n'est pas difficile de pointer toutes les fois où, ensemble, nous avons rencontré la "chose noire», nous en approchant et nous en éloignant, entre souffles et soupirs. Dans ce genre de dialogues, reprendre haleine n'est pas moins important que le fait même de parler. Par ailleurs, la rencontre avec la « chose » n'a pu avoir lieu qu'après l'échec de la biomédecine, qui a laissé Hannah littéralement sans voix. C'est l'absence de diagnostic qui nous impose, devant la persistance du symptôme, de chercher d'autres interprétations, nettement plus angoissantes. Le médecin de l'hôpital avait rassuré la jeune femme en la renvoyant chez elle avec un vague «tu n'as rien, ne t'en fais pas ». Mais Hannah ne pouvait pas ne pas s'en faire et, de plus, elle ne voulait pas mourir empoisonnée.

Dans la sorcellerie, parler est une façon contrariée de se souvenir - le temps à accorder à un non pour qu'il devienne un oui -, mais aussi un appel à l'aide ambivalent, adressé directement à son interlocuteur. Qu'est-ce que tu en sais? Et surtout, qu'est-ce que tu peux, toi, pour moi ?

Depuis Jeanne Favret-Saada (1977), les études anthropologiques sur la sorcellerie ont acquis la complexité d'un savoir-pouvoir agissant sur la réalité, qui implique directement le désir du chercheur et l'entraîne à prendre position. Les conséquences pour la recherche ne sont pas moins insidieuses lorsqu'on s'adresse non plus à ceux qui savent, mais à ceux qui craignent, soupçonnent, sont en quête d'une réponse au sujet de leur mal-être. Lorsqu'on écoute les personnes ayant le sentiment d'avoir été agressées et qui « savent» dans leur propre corps la sensation de la chute dans l'angoisse, il est difficile de ne pas être impliqué, de rester en dehors d'une relation qui est déjà, dès lors qu'on la formule, une forme d'action. On est poussé à faire quelque chose pour modifier l'état des choses, en arrivant au moins à réduire l'angoisse mortelle qui se dégage intensément des dires et du corps de l'autre.

Au cours de ces recherches, on est évidemment exposé à des rencontres nombreuses et hétérogènes avec des "choses» et des rumeurs difficiles à interpréter. Mais certains événements ne trompent pas.

commerçante à Onitsha et Enugu pour y acheter des produits à revendre au village (entretien avec F.I., avril 2010, Turin). 
[E]ntre le sorcier et les récits qui circulent à l'envi, il existe un élément intermédiaire et tangible. C'est la crise sorcellaire qui frappe un sujet, l'atteint dans son corps ou ses biens, provoque dans son entourage proche un déferlement d'angoisse, voire de panique, et déclenche une séquence où des conduites corporelles [...] se conjuguent à des paroles performatives, à des énonciations et à l'usage de substances et d'objets matériels dans la cure. C'est l'événement qui nous livre l'image spéculaire de l'insaisissable sorcier (Warnier 2017 : 129).

Lorsqu'on est confronté à la crise sorcellaire, il est impossible de ne pas la reconnaître : elle emporte tout et tout un chacun vers un champ de bataille et de choix à faire. Nous y sommes aussi impliqués, nous autres anthropologues, en fonction de rythmes et de modalités certes disparates, selon des variables professionnelles et personnelles, mais sans issue par rapport aux demandes pressantes de nos interlocuteurs. Pour preuve, Matilda Coxe Stevenson qui, au cours de l'expédition ethnologique américaine chez les Zunis au début du $\mathrm{XX}^{\mathrm{e}}$ siècle, confrontée au jeune homme de dix-sept ans accusé de sorcellerie, décida de l'emmener définitivement avec elle loin du village où il avait grandi ${ }^{22}$; de son côté, Jean-Pierre Warnier nous raconte honnêtement qu'en 1983, pendant quelques semaines, pour répondre à une requête d'un de ses collègues universitaires camerounais attaqué par la sorcellerie, il fit le trajet entre l'université et le hameau du tradipraticien de façon à lui apporter les mémoires des étudiants à corriger. Le fait d'être étranger ne protège pas toujours des accusations. Nous nous souvenons tous de l'écriture prudente du père Éric de Rosny (1981) à ce sujet, lorsqu'il aborde l'hypothèse malaisée qu'un prêtre puisse donner la mort à quelqu'un...

On réagit donc aux demandes de l'autre qui est ensorcelé, on ne peut pas rester les bras ballants. Chacun peut sans aucun doute témoigner, d'après ses carnets, ses pensées et ses expériences, d'épisodes semblables. J'aimerais ici suggérer que ces élans vers l'action (et vers la réaction) ne sont pas uniquement le fait de ceux qui opèrent dans le cadre des soins : dit d'une autre façon, je laisse entendre ici que la sorcellerie est une croyance ayant pour effet étrange de faire de chacun un acteur potentiel de la cure, car elle modifie la façon d'écouter l'autre au moment où la « chose » se manifeste bien au-delà d'une simple représentation inoffensive.

Il y a bien sûr des façons de se démarquer par rapport à ce genre de relations visqueuses : en tout premier lieu, revendiquer notre extranéité en concluant prématurément que ce dont parlent Hannah, le jeune Zuni, Jean ou Din «ne peut pas réellement exister». Jeanne Favret-Saada (1977: 37) n’avait pas

22. L'intervention de la chercheuse fut très violente à l'égard de la communauté du jeune homme et du conseil des notables, qu'elle fit arrêter dès qu'elle eut quitté le village (SIEGEL 2006). 
lésiné sur les critiques envers cette démarche ethnographique : «Prétendre [...] qu'on veut entendre parler de sorcellerie paysanne et y rester étranger, c'est se condamner à n'entendre que des déclarations objectivistes, à collectionner des historiettes fantastiques et des recettes de désenvoûtement — soit, à relever des énoncés que le sujet de l'énonciation désavoue formellement. »

Mais je pense ici plutôt à un débat beaucoup plus récent, qu'Eduardo Viveiros de Castro (2015) et David Graeber (2015) ont entretenu à distance. Viveiros de Castro reprochait à Graeber d'avoir présenté une « description défectueuse » du monde magique mérina et du pouvoir de certains de leurs objets parce qu' « on avait décidé dès le début » (comme auraient dit Deleuze \& Guattari [1980]) que, tels que les concevaient les Mérina, les pouvoirs magiques et les fétiches ne pouvaient pas exister réellement (" cannot really exist ») $)^{23}$. Une « description suffisamment bonne » aurait dû, selon Viveiros de Castro, laisser une issue de secours aux Mérina en termes d'autodétermination de la réalité.

Nous arrivons sur le terrain pour faire de la recherche. Nous rencontrons des personnes qui deviennent nos interlocuteurs. Si nous décidons dès le début ce qui « peut réellement exister », n'importe quelle aspiration anthropologique à décrire le social ne perd-elle pas tout son sens? Et encore : penser que le pouvoir magique mérina, tel qu'il est conçu par les Mérina (même si des interprétations du mal d'une autre nature coexistent) ne peut vraiment exister ne revient-il pas, de fait, à décréter déjà de façon univoque et cristalline ce qui ne nous fait pas peur?

L'espace ethnographique se restreint et on risque le dérapage. Un pas de trop et nous nous engouffrons dans un tourbillon d'accusations et d'excuses. Un pas en arrière et nous rentrons chez nous qu'avec des historiettes. Si nous décidons de suivre notre interlocuteur dans ses peurs les plus intimes et de répondre à sa demande de faire quelque chose, nous nous exposons inévitablement à des risques méthodologiques : de la réification à l'aveuglement; de la contagion à la suggestion, au relativisme. Personnellement, je pense que les anthropologues doivent bien courir quelques-uns de ces risques, car c'est la seule façon de sortir de l'effervescence de la représentation au détriment de la chose.

23. " Of course it would also be going too far to say that the fetishistic view is simply true : Lunkanka cannot really tie anyone's intestines into knots; Ravololona cannot really prevent hail from falling on anyone's crops » (Graeber cité dans VIVEIROS DE CASTRO 2015 : 15 ; nos italiques). En relisant ces travaux à la lumière des dernières réflexions livrées par WARNIER (2017), on pourrait penser que ce risque se produit lorsqu'on travaille sur des récits, des narrations, des rumeurs et des bavardages, bref sur la « représentation sorcellaire » et non sur la « crise ». Voir aussi la réponse de GrAEBER (2015). 
Traces pour une sorcellerie des fesses

Si l'on passe maintenant en revue l'ethnographie présentée ici, il apparait clairement que ce système migratoire expose à des crises répétées, qu'il est pensé pour les produire et reproduire sans cesse. Faire de la recherche veut dire en premier lieu s'exposer au rôle qui nous est attribué par nos interlocutrices, dès lors qu'elles sentent leur vie s'évanouir. La «mère de derrière » est l'agresseur agissant à travers l'organe de contrôle de sa propre « fille». Chaque jeune femme doit avoir au moins une mère de derrière, pour partir, et chaque mère de derrière doit avoir un ou plusieurs organes de contrôle externe de ses filles. Que ce soit la mère de l'anus au sens littéral ${ }^{24}$ ou la mère de derrière au sens figuré, c'est en position d'agresseur que cette femme parle et agit, grâce à un organe externe resté dans ses mains (où dans des mains des personnes alliées).

Si on suit l'indice fourni par Patrice Yengo, on parvient à saisir le sens de cette arrière-scène et à comprendre pourquoi, dans ce monde-là, la mort ne peut l'être que si elle est pensée comme annoncée.

[La] dimension spatiale [de la sorcellerie] peut être référencée dans la césure entre l'avant et l'arrière, devant et derrière, du fait que la [sorcellerie] est le domaine des arrière-pensées et que les attaques en sorcellerie ne sont donc jamais frontales. Provenant toujours de derrière, elles nécessitent toujours une vision de derrière (Yengo 2016 : 59).

Une autre figure topologique de l'inversion, reliée à la sorcellerie, est celle de l'envers et l'endroit. Si André Mary (1988) notamment la développe pour décrire le rituel bwiti fang de la «naissance à l'envers », pouvons-nous penser à la substance ensorcelante comme étant capable de naître à l'envers ? C'est un regressus ad uterum bien particulier qu'accomplit, par exemple, l'evu au Gabon et au Cameroun, en pénétrant dans le vagin de la femme pour aller se positionner dans son ventre, pour y rester sans la moindre intention de venir au monde. Son existence réside dans la régression, sans jamais plus en sortir. Or, dans la diaspora criminelle nigériane (et peut-être bien au-delà de celle-ci),

24. «Iye onisan » est littéralement la « mère de l'anus » comme « iye omo » signifie « mère de l'enfant» (par exemple, «iye Oghogho» est la «mère de Oghogo »: faire suivre le terme "iye » du nom du fils aîné est la façon dont les mères sont appelées après l'accouchement, preuve d'un changement de statut social en devenant mères). Comme dans d'autres contextes sociaux, on ne s'adresse plus aux femmes dans l'espace public par leur propre nom, mais on les appelle «mères de ». Or, "iye onisan » pourrait donc bien être la «mère de » l'anus ou du cul/trou, organe associé au pouvoir sorcellaire dans différents contextes de l'Afrique subsaharienne. Voir le travail d'A. CERIANA MAYNeri (2014 : 11) sur l'« anus de sorciers » en Centrafrique. 
nous sommes au sein d'un système de relations humaines qui nous obligent à (re)penser au rapport entre orifices, contenants et morceaux d'organes dans la sorcellerie. Non pas l'utérus, mais l'anus. Non pas un enfant qui voit le jour ou bien un être qui se cache dans le bas-ventre, mais des morceaux-de-filles produits par des déchets et des restes de leurs corps éparpillés un peu partout dans le monde. Je ne prétends pas développer davantage ces associations, mais je reconnais en elles les ingrédients d'une ethnopsychologie de l'abondance qui peut rapidement précipiter en une psychologie de la résignation et du renoncement, à partir de la transformation de n'importe quelle partie du corps (salivation, chaleur, fourmillement, douleur, etc.).

En conclusion, j'aimerais reprendre dans ses grandes lignes ce que Deleuze \& Guattari (1972) écrivaient sur l'investissement collectif des organes dans la machine sociale précapitaliste. Selon eux, ces sociétés célébraient des « organes-objets partiels » d'où se détachaient, à côté des vagins cloués sur le corps des femmes et des pénis immenses partagés entre les hommes, «l'anus indépendant» et « le corps sans anus » (il ne nous est cependant pas donné de savoir à quel mythe se réfèrent les auteurs).

Nos sociétés modernes [...] ont procédé à une vaste privatisation des organes, qui correspond au décodage des flux devenus abstraits. Le premier organe à être privatisé, mis hors champ social, fut l'anus. C'est lui qui donna son modèle à la privatisation, en même temps que l'argent exprimait le nouvel état d'abstraction des flux (ibid. : 168).

Est-ce qu'on peut expliquer l'expansion de cette sorcellerie de fesses pratiquée par des mères-anus - complexe sorcellaire conçu ici avant tout comme un certain rapport au temps (donc à une mort qui ne pourrait plus être considérée comme accidentelle) du subalterne, de l'opprimé, de l'obéissant - comme une forme de ce qui reste inviolable dans l'expérience de soi, la conscience du monde, la perception de la réalité, la réalité elle-même ? Est-ce que la rencontre ethnographique elle-même ne peut pas déjà être une relation en quête d'une voie de sortie dans un chemin qui n'est pas choisi par le chercheur? Pourquoi privatiser trop rapidement les organes, que ce genre de migration semble exposer sans aucune pudeur à l'investissement collectif (et à une jouissance économique certaine) ? Et enfin, la «magification» du monde (à ne pas confondre avec ce qu'on appelle génériquement « renaissance du religieux » ou avec sa pentecôtisation) peut-elle recéler un refus obstiné de mourir? 
S'il est vrai que la magie et la sorcellerie sont, au sens large, un champ de communication rapprochée - c'est-à-dire privée de toute transcendance entre les hommes et les esprits, alors le monde «désenchanté » des monothéismes et de la science est en conflit ouvert avec le monde magique, qui permet quelque chose que la toute-puissance du dieu blanc n'offre plus et le savoir scientifique n'a jamais offert: la possibilité de négocier avec les instances qui nous contrôlent. Le désenchantement correspond donc non pas à un surplus de pouvoir, mais à une perte de marge de négociation. Peut-être est-ce aussi pour cela que les femmes nigérianes que je rencontre sont toujours en quête d'un monde magique et sorcellaire dès lors que la crise se manifeste dans la migration, le seul qui leur permette de s'en sortir en négociant, même avec moi, des « hypothèses de vie qui élargissent leur vie $»^{25}$.

Université de Turin, Département de cultures, politique et société, Turin (Italie).

\author{
BIBLIOGRAPHIE
}

Aderinto S., 2015, «Journey to Work: Transnational Prostitution in Colonial British West Africa », Journal of the History of Sexuality, 24 (1) : 99-124.

AdLer A., 2002, «Royauté, magie et religion. "Faire dialoguer de manière intime anthropologie et histoire" », L'Homme, 163 : 173-183.

Agheysi N. R., 1986, An Edo-English Dictionary, Benin City, Ethiope.

Ahiaba M., 2015, «Retrieving Ebo as Spirit: The Foundation of Authentic Christian Pneumatology among the Igala, Nigeria », Cross-Cultural Communication, 11 (3) : 7-19.

BAYART J.-F., 2014, « À nouvelles pratiques religieuses, nouveaux instruments d'analyse? L'écriture abiographique des plans de foi », in P. Chanson, Y. Droz, N. Y. Gez \& E. SoARes (dir.), Mobilité religieuse. Retours croisés des Afriques aux Amériques, Paris, Karthala : 39-52.

BeRnaUlt F., 2009, «De la modernité comme impuissance. Fétichisme et crise du politique en Afrique équatoriale et ailleurs », Cahiers d'Études africaines, XLIX, 195 (3) : 747-774.

25. Je reprends ici l'expression de SAnsot (2015) dans son Paysages de l'existence. 
Bonhomme J., 2005, « Voir par-derrière. Sorcellerie, initiation et perception au Gabon », Social Anthropology, $13: 259-273$.

Bradbury R. E., 2017, The Benin Kingdom and the Edo-speaking Peoples of SouthWestern Nigeria, New York, Routledge.

Ceriana Mayneri A., 2014, Sorcellerie et prophétisme en Centrafrique : l'imaginaire de la dépossession en pays banda, Paris, Karthala.

DE CERTEAU M., 1981, «Une pratique sociale de la différence : croire », numéro thématique, «Faire croire. Modalités de la diffusion et de la réception des messages religieux du $\mathrm{XII}^{\mathrm{e}}$ au XV $\mathrm{e}^{\mathrm{e}}$ siècle », Actes de table ronde de Rome, 22-23 juin 1979, 51 (1) : 363-383.

Claverie É., 2015, «Vivre dans le “combattantisme”. Parcours d'un chef de milice en Ituri (République démocratique du Congo)», Terrain. Anthropologie et Sciences humaines, $65: 159-181$.

Deleuze G. \& Guattari F., 1972, L’Anti-CEdipe. Capitalisme et schizophrénie, Paris, Les Éditions de Minuit.

— 1980, «Traité de nomadologie. Machine de guerre», in Capitalisme et schizophrénie 2. Mille plateaux, Paris, Les Éditions de Minuit : 434-527.

Dezalay S., 2017, «L'Afrique contre la Cour pénale internationale ? Éléments de sociogenèse sur les possibles de la justice internationale », Politique africaine, 146 : 165-182.

EkeH P. P., 1975, «Colonialism and the Two Publics in Africa : A Theoretical Statement », Comparative Studies in Society and History, 17 (1) : 91-112.

Ellis S., 2016, This Present Darkness : A History of Nigerian Organized Crime, New York, Oxford University Press.

FAVRet-SaAda J., 1977, Les mots, la mort, les sorts, Paris, Gallimard.

Fourchard L., 2013, «Prêt sur gage et traite des femmes au Nigeria, fin XIXe-années 1950 », in B. LAVAud-Legendre (dir.), Prostitution nigériane: entre rêves de migration et réalité de la traite, Paris, Karthala : 13-30.

George A., 2014, Making Modern Girls. A History of Girlhood, Labor, and Social Development in Colonial Lagos, Athens, Ohio University Press.

Geschiere P., 2013, Witchcraft, Intimacy, and Trust : Africa in Comparison, ChicagoLondon, University of Chicago Press.

GrAeBer D., 2015, « Radical Alterity Is Just Another Way of Saying "Reality". A Reply to Eduardo Viveiros de Castro », Hau : Journal of Ethnographic Theory, 5 (2) : 1-41.

De Heusch L., 2000, Le Roi de Kongo et les monstres sacrés, Paris, Gallimard.

LaVAud-Legendre B. (DIR.), 2013, Prostitution nigériane : entre rêves de migration et réalité de la traite, Paris, Karthala. 
MARY A., 1988, «Le schème de la naissance à l'envers : scénario initiatique et logique de l'inversion », Cahiers d'Études africaines, XXVIII, 110 (2) : 233-263.

Melzian H., 1937, A Concise Dictionary of the Bini Language of Southern Nigeria, London, Kegal Paul.

NAanen B. B. B., 1991, «"Itinerant Gold Mines” : Prostitution in the Cross River Basin of Nigeria, 1930-1950», African Studies Review, 34 (2) : 57-79.

Nodal R. \& Ramos M. W., 2005, «Let the Power Flow : Ebó as a Healing Mechanism in Lukumí Orisha Worship », in P. Bellegarde-Smith (ed.), Fragments of Bone. Neo-African Religious in a New World, Urbana-Chicago, University of Illinois Press : 167-186.

Northcote W. T., 2010 [1910], Anthropological Report on the Edo-speaking Peoples of Nigeria, London, Harrison \& Sons, Internet Archive, University of Toronto, $<$ https://archive.org/stream/anthropologicalr00thom/anthropologicalr00thom_djvu.txt>.

Ortigues M. C. \& Ortigues E., 1984, L'Edipe africain, Paris, L'Harmattan.

PIETz W., 2005, Le fétiche : généalogie d'un problème, Paris, Kargo-l'Éclat.

Pratten D., 2007, The Man-Leopard Murders : History and Society in Colonial Nigeria, Edinburgh, Edinburgh University Press.

DE Rosny É. (DIR.), 1981, Les yeux de ma chèvre, Paris, Plon.

— 2006, Justice et sorcellerie, Paris, Karthala.

SAnsot P., 2015, Paysages de l'existence, Gollion, InFolio, Archigraphy.

Siegel T. J., 2006, Naming the Witch, Stanford, Stanford University Press.

Tацвот P. A., 1967, Tribes of the Niger Delta: Their Religions and Customs, London, Frank Cass.

TALIANi S., 2012, « Coercion, Fetishes and Suffering in the Daily Lives of Young Nigerian Women in Italy », Africa, 82 (4) : 579-608.

— 2016, «Calembour de choses dans le vaudou italien : Corps-fétiche et principes d'inégalité devant les dieux », Social Compass, 63 (2) : 163-180.

— 2017, «Femmes nigérianes déplacées, filles à la merci. Sur les usages de l'ethnopsychiatrie », in A. CERIANA MAYNERI (dir.), Entre errances et silences. Ethnographier des souffrances et des violences ordinaires, Louvain-la-Neuve, Academia-L'Harmattan : 65-98.

Tonda J., 2015, L’impérialisme postcolonial. Critique de la société des éblouissements, Paris, Karthala (« Les Afriques »).

Viveiros de Castro E., 2015, « Who is Afraid of the Ontological Wolf? Some Comments on an Ongoing Anthropological Debate », The Cambridge Journal of Anthropology, $33(1): 2-17$. 
WARNIER J.-P., 1988, «L'économie politique de la sorcellerie en Afrique centrale », Revue de l'Institut de sociologie, 3-4 : 259-271.

— 2017, «Ceci n'est pas un sorcier. De l'effet Magritte en sorcellerie », Politique africaine, $146: 125-141$.

WiLKInson J. (ED.), 1992, Talking with African Writers. Interviews with African Poets, Playwrights and Novelists, London, James Currey.

Yengo P., 2016, Les mutations sorcières dans le bassin du Congo. Du ventre et de sa politique, Paris Karthala.

RÉSUMÉ

La migration théocratique nigériane vers l'Europe s'appuie sur une psychologie spirituelle de l'abondance qui, dans le cas de la traite, implique habituellement deux femmes: l'une, plus âgée, qui dispose des moyens financiers permettant d'assumer tous les frais du voyage de l'autre, à qui elle indique les personnes auxquelles s'adresser au cours des nombreuses étapes du trajet; et la cadette qui, une fois arrivée dans un pays européen, utilisera son corps comme un capital à exploiter pour rembourser sa dette. Entre ces deux protagonistes « de la nuit» s'instaure une dépendance fondée sur une action rituelle destinée à signifier une parenté fictive, sur l'existence d'un organe de contrôle et sur le serment prêté à l'un des « deux-cent-et-un » esprits du panthéon edo. Cette scène migratoire est à l'origine d'un complexe sorcellaire dont la crise de la cadette constitue une des manifestations névralgiques les plus fréquentes, où la figure de la «mère de derrière » incarne l'agresseur familial et devient signe avant-coureur d'une malédiction attendue.

Mots-clés: Benin City, Turin, cadets sociaux, crise sorcellaire, dette, diaspora nigériane, fétiches, organe de contrôle, relation mère-filles, traite des êtres humains.

\section{ABSTRACT}

On the Predicament of the Daughters and the Resources of Life. Witchcraft Crisis in Nigerian Migration. - The Nigerian theocratic migration to Europe relies on a spiritual psychology of abundance that within the human trafficking system involves two women. There is an older woman who has the financial resources to bear the travel costs of the younger one and who gives her the contacts she needs for her long journey and to find work and a younger woman who, upon arrival in Europe, will use her own body as a capital to exploit in order to reimburse her debt. Between these two protagonists "of the night" a dependent relationship is founded on a ritual intended to signify a fictive kinship, on the existence of an external controlling organ and on a vow made to one of the "twohundred-and-one" spirits of the Edo pantheon. This migration scene is situated at the origin of a witchcraft complex in which the young woman's crisis constitutes one of its principle and most frequent manifestations where the figure of the "mother-buttocks" embodies the family aggressor and becomes the sign foreshadowing the advent of an expected curse.

Keywords: Benin City, Turin, debt, external controlling organ, fetishes, human trafficking, mother-daughter relationship, social cadets, witchcraft crisis. 